\title{
1 MetaCompass: Reference-guided Assembly of Metagenomes
}

2 Victoria Cepeda ${ }^{1,2,+}$, Bo Liu ${ }^{1,2,+}$, Mathieu Almeida ${ }^{2}$, Christopher M. Hill ${ }^{1,2}$, Sergey Koren ${ }^{3}$,

3 Todd J. Treangen ${ }^{2}$, Mihai Pop ${ }^{1,2 *}$

$5 \quad{ }^{1}$ Department of Computer Science, University of Maryland, College Park, Maryland, USA.

$6{ }^{2}$ Center for Bioinformatics and Computational Biology, University of Maryland, College Park, 7 Maryland, USA.

$8 \quad{ }^{3}$ Genome Informatics Section, Computational and Statistical Genomics Branch, National Human

9 Genome Research Institute, Bethesda, Maryland, USA.

$10+$ These authors contributed equally to this work

$11 *$ mpop@umd.edu, to whom correspondence should be addressed

\section{ABSTRACT}

14 Metagenomic studies have primarily relied on de novo approaches for reconstructing genes and

15 genomes from microbial mixtures. While database driven approaches have been employed in certain

16 analyses, they have not been used in the assembly of metagenomes. Here we describe the first

17 effective approach for reference-guided metagenomic assembly of low-abundance bacterial genomes

18 that can complement and improve upon de novo metagenomic assembly methods. When combined

19 with de novo assembly approaches, we show that MetaCompass can generate more complete 
assemblies than can be obtained by de novo assembly alone, and improve on assemblies from the

21 Human Microbiome Project (over 2,000 samples).

23 Keywords: metagenome assembly, microbiome, low coverage assembly, comparative assembly

\section{Background}

25 Microorganisms play an important role in virtually all of the Earth's ecosystems, and are critical for

26 the health of humans [1], plants, and animals. Most microbes, however, cannot be easily grown in a

27 laboratory [2]. The analysis of organismal DNA sequences obtained directly from an environmental

28 sample (a field termed metagenomics), enables the study of microorganisms that are not easily

29 cultured. Metagenomic studies have exploded in recent years due to the increased availability of

30 inexpensive high-throughput sequencing technologies. For example, the MetaHIT consortium

31 generated about 500 billion raw sequences from 124 human gut samples in its initial analysis [3], and

32 the Human Microbiome Project (HMP) has generated hundreds of reference microbial genomes and

33 thousands of whole metagenome sequence datasets from healthy subjects [4].

35 The analysis of these vast amounts of data is complicated by the fact that reconstructing large

36 genomic segments from metagenomic reads is a formidable computational challenge. Even for single

37 organisms, the assembly of genome sequences from short reads is a complex task, primarily due to

38 ambiguities in the reconstruction that are caused by genomic repeats [5]. In addition, metagenomic

39 assemblers must be tolerant of non-uniform representation of genomes in a sample as well as of the

40 genomic variants between the sequences of closely related organisms. Despite advances in 
41 metagenomic assembly algorithms over the past years [6-10], the computational difficulty of the assembly process remains high and the quality of the resulting assemblies requires improvement.

Consequently, many analyses of metagenomic data are performed directly on unassembled reads [1115], however the much shorter genomic context leads to lower accuracy [16].The need for effective and efficient metagenomic assembly approaches remains high, particularly since long read technologies (which partly mitigate the challenges posed by repeats [17-19]) are not yet effective in metagenomic applications due to lower throughput, higher costs [20,21], and higher required DNA quality and concentration.

Reference-guided, comparative assembly approaches have previously been used to assist the assembly of short reads when a closely related reference genome was available [22, 23]. Comparative assembly works as follows: short sequencing reads are aligned to a reference genome of a closely related species, then their reconstruction into contigs is inferred from their relative locations in the reference genome [23]. This process overcomes, in part, the challenge posed by repeats as the entire read (not just the segment that overlaps within adjacent reads) provides information about its location

57 in the genome.

Currently, thousands of bacterial genomes have been sequenced and finished [24], and this number is expected to grow rapidly soon thanks to long read technologies. These sequenced genomes provide a

61 great resource for performing comparative assembly of metagenomic sequences. Comparative

62 approaches developed in the context of single genomes cannot, however, be directly used in a 63 metagenomic setting. Simply mapping a set of reads to even hundreds of different genomes is 
currently computationally prohibitive. Furthermore, genome databases comprise many variants of a same genome (e.g., the US FDAs GenomeTrackr project [25] alone has contributed over 60,000 different strains of Salmonella), and genome by genome analyses would result in redundant reconstructions of metagenomic sequences. We also note that some recent reference-guided strategies implemented in genomic analysis tools, such as the "--trusted-contigs" feature of the SPAdes assembler [26, 27] and StrainPhlan [28] ignore the fact that the data being reconstructed originates from genomes that are related but different from the genomes found in public databases. As a result, such approaches may actually mis-assemble the metagenomic data exactly within the genomic regions where novel biological signals may be located.

In this paper, we describe the first effective assembly software package for the reference-assisted assembly of metagenomic data. We rely on an indexing strategy to quickly construct sample-specific reference collections, thereby dramatically reducing the computational costs of mapping metagenomic reads to references. Furthermore, we eliminate redundancy in the assembly by disambiguating the mapping of reads against closely related genomes, and identify differences between the metagenomic data and the reference genomes in order to reduce the likelihood of misassembly.

We show that our approach effectively complements de novo assembly methods. We also show that the combination of comparative and de novo assembly approaches can boost the contiguity and completeness of metagenomic assembly, and provide an improved assembly of the entire wholemetagenome sequencing data generated by the Human Microbiome Project [4]. 
87 Our software is released freely under an open-source license at: http://www.github.com/marb1/MetaCompass .

\section{Results}

All assemblies were compared based on contiguity statistics, number of errors, and based on the number of complete phylogenetic marker genes found in the final assembly - a measure of how useful an assembly may be to downstream analyses. The coverage of the set of marker genes has been used by the HMP and others $[3,29,30]$ as measure of the completeness of an assembly.

\section{Evaluation of performance on synthetic metagenomic dataset}

We first evaluated MetaCompass by assembling a synthetic microbial community [31]. The synthetic sample was downloaded from the NCBI Short Read Archive (SRA) database, (SRR606249) and contains 54 bacteria and 10 archaea. Among these organisms, 55 had complete genome sequences in the NCBI RefSeq database (the database used by default by MetaCompass), and 9 were available only as a high-quality draft assembly at the time of publication. Since the true genome sequences are known, these data are ideal as they allow us to fully quantify the quality of the genomic reconstruction.

We set the minimum coverage in MetaCompass at 1-and 2-fold (see Methods), then performed reference genome selection (see Methods and Supplementary Table 1). The assembly results (Table 1, see MetaCompass $1 \mathrm{X}$ and $2 \mathrm{X}$ ) can be considered an approximate upper bound on the performance of any assembly tool, as in this case $90 \%$ of the genomes recruited were exactly those from which the metagenomic reads were obtained. We compared the performance of MetaCompass with that of three widely used de novo assemblers: IDBA-UD (July 2016) [8], MEGAHIT (v1.0.6) [32], and 
109 metaSPAdes (v3.9.0) [33]. Compared with these assemblers, MetaCompass achieved higher genome

110 recovery (Table 1, Figure 1) and produced significantly larger and more accurate contigs (Table 1).

111 When we decreased the MetaCompass minimum coverage threshold from 2-fold to 1-fold, we

112 observed gains in maximum contig size and total aligned length, while retaining a similar error

113 profile. However, we observe higher genome recovery at minimum coverage threshold 2 and 3 . On

114 the basis of the maximum contig size, total aligned length, error profile and genome recovery, we

115 chose $2 \mathrm{X}$ as default setting for MetaCompass. Note that here we are not trying to prove that

116 MetaCompass is better than de novo assemblers, and in this setting, the comparison is not fair

117 because our reference collection contains the exact genomes present in the samples. Rather, we are

118 trying to show that the performance of MetaCompass can be excellent if the reference collection

119 contains genomes highly similar to those in the metagenomic sample being assembled.

\section{References removed from database}

121 To provide a better idea of how MetaCompass would perform in a worst-case scenario, we removed

122 from the database the genomes represented in the mock community (Supplementary Table 2), thereby

123 forcing MetaCompass to recruit near-neighbor reference genomes, when available. (see

124 'MetaCompass.nr' row, Table 1). Median genome recovery for MetaCompass is just $1 \%$ less than

125 that of de novo assemblers. The accuracy of the reconstruction, as measured by mismatch and indel

126 rates, is higher than that of IDBA-UD and metaSPAdes (Table 1, MetaCompass.nr (2x)), while

127 moderately lower than MEGAHIT. 
128 The number of misassemblies and local misassemblies per $1 \mathrm{Mbp}$ of assembled sequence (as reported

129 by MetaQuast [34]) increased from 2.0 to 4.9 when reducing the coverage threshold to 1 . To put this

130 increase into context, we measured the total number of possible errors by evaluating the "accuracy"

131 of the near-neighbor reference genomes recruited by MetaCompass with respect to the correct

132 reference sequence (Figure 2, see hashed blue bar). This allows us to capture the real differences

133 between the recruited reference genomes and the actual genome represented in the synthetic dataset

134 [31], essentially providing an upper bound for the number of errors MetaCompass would make if it

135 simply recapitulated the sequence of the selected reference genomes. As seen in Figure 2,

136 MetaCompass is making five times fewer errors than would be expected, indicating our software is

137 not unduly biased by the sequence and structure of the reference genome.

\section{Evaluation of performance on downsampled synthetic datasets}

139 To evaluate the ability of MetaCompass to assemble low-coverage genomes, we down-sampled the

140 synthetic dataset to just 5 million paired-end reads, or $10 \%$ of the original data set. After down-

141 sampling, the average coverage was reduced to approximately 3-fold (data not shown). The results

142 (Table 2, Figure 3) highlight that MetaCompass can recover a median of 90\% of each of the 64

143 genomes in the sample. While metaSPAdes comes in second place and is able to recover $80 \%$

144 (median recovery), it does so at the cost of a four times higher mis-assembly rate. The two remaining

145 methods, MEGAHIT and IDBA-UD, leave a quarter to a half of the genomes unassembled (Table 3).

\section{Computational performance}

147 When dealing with large-scale data sets, the combination of total required memory and run time is an

148 important factor in determining the applicability of a computational tool. We first evaluated the

149 runtime performance of MetaCompass on a Linux 12-core server node with 80 GB of memory using

150 the Shakya et al. synthetic dataset. The wall clock run time on this synthetic dataset for MetaCompass 
151 is comparable to that of de novo assemblers, sometimes lower (Supplementary Table 3).

152 MetaCompass (without PILON) and Megahit were the only approaches that required <16GB of RAM

153 on a 100 million read dataset, highlighting the scalability of these methods to large datasets.

\section{Reassembly of the data generated by the Human Microbiome Project (HMP2)}

155 To further explore the benefits and limits of comparative approaches for metagenomic assembly, we

156 re-analyzed with MetaCompass 2,077 metagenomic samples from the HMP Project (ftp://public-

157 ftp.hmpdacc.org/Illumina/PHASEII/). These samples cover 15 body sites from four broad regions of

158 the human body: oral, skin, stool, and vaginal. We compared the assemblies produced by

159 MetaCompass with the official IDBA-UD assemblies reported by the HMP project [35]. Note that

160 these assemblies were recently improved by Lloyd-Price et al. [36] but we did not include them in

161 this study. Across all samples, on average, MetaCompass outperforms the HMP2 de novo approach,

162 leading to an overall better assembly of the original data (Table 3, Figure 4). However, the relative

163 performance of MetaCompass and the HMP2 assembly varied across body-sites due to the specific

164 characteristics of the microbial communities being reconstructed. While MetaCompass generates

165 more assembled sequence and complete marker genes across all body sites, the maximum contig size

166 and size at $1 \mathrm{Mbp}$ metrics vary per body site. In oral and stool samples (Figure 4), MetaCompass

167 outperforms de novo assembly for all metrics. In skin and vaginal samples (Figure 4), the de novo

168 approach has better contiguity statistics but MetaCompass assembles more complete marker genes.

169 To gain further insight into these results we calculated the average nucleotide identity between the de

170 novo assembled contigs and the recruited reference genomes for each body site. In all body sites,

171 except for oral, the assembled contigs had 99\% average nucleotide identity to the reference genomes.

172 In the oral samples, the most distant reference genomes had only $97 \%$ identity to the assembled

173 contigs, indicating that at least in part, the lower effectiveness of MetaCompass is due to the absence

174 of a sufficiently closely related reference genome for some of the oral samples. 
176 To further explore the drop in contiguity in skin and vaginal samples, we focused on just the contigs

177 that mapped to bacterial genomes contained in the reference database, allowing for a direct

178 comparison between MetaCompass and de novo contigs. The results shown in Table 4 indicate that

179 for this set of contigs, MetaCompass outperforms the de novo approach for the vaginal samples.

180 However, the de novo HMP2 assembly of the skin sample is still better in terms of complete genes

181 recovered, but equivalent to MetaCompass with respect to complete marker genes recovered (a

182 measure of assembly completeness).

183 Comparing reference-guided to de novo assembly on low-coverage HMP2 samples

184 To assess the ability of MetaCompass to assemble low-abundance organisms, we focused on all skin

185 HMP2 samples. The skin samples had the second lowest average number of reads while still

186 containing reasonable diversity and richness, as reported in Table 3 . We removed the contigs

187 assembled via de novo assembly from the MetaCompass output, collected the reference genomes that

188 were used, mapped the HMP2 contigs to these reference genomes, and then evaluated the number of

189 complete genes and complete marker genes in both. Compared to the HMP2 assembly, reference-

190 guided assembly of these low coverage samples is able to reconstruct approximately $10 \%$ more

191 marker genes $(4,423$ versus 3,915$)$ than the de novo approach, roughly equating to 10 additional

192 complete bacterial genomes in total.

193

194 We next searched for microbes that were present in the skin samples at relatively low coverage and

195 explored the differences between the reconstructions generated by the HMP2 project and

196 MetaCompass. Specifically, we identified a low coverage assembly of a Propionibacterium acnes

197 genome reconstructed by both MetaCompass and the HMP in sample SRS057083. The HMP2 
198

199

200

\section{Discussion}

202

203

204

205

206

207

208

209

210

211

212

213

214

215

216

217 While MetaCompass provided an advantage over de novo approaches for most of the human-

218 associated microbial communities sampled by the HMP project, in skin samples the performance of

219 MetaCompass was on average lower than the assemblies produced by the HMP. This result could be 
220 due to structural genome dynamics of bacterial defense systems commonly found in skin microbes

221 [37-39], situation that introduces frequent structural variants between the reference genomes and the

222 corresponding environmental isolates. We plan to further explore this hypothesis through graph-

223 based analyses of de novo assemblies of the corresponding communities.

MetaCompass relies on the taxonomic profiling tool MetaPhyler as an efficient indexing strategy for

226 identifying the reference genomes most closely related to the data being assembled. Compared to

227 whole-genome indices, the MetaPhyler index is based on just 18 phylogenetic marker genes that are

228 ubiquitous in bacteria, thus providing a compact and efficient data-structure. Using marker genes

229 ensures that any genome present at a high enough coverage to allow assembly will be detected

230 despite indexing just a small fraction of its genome. Since MetaPhyler, and other similar tools [40,

$23141]$ are designed for much broader use cases than those targeted here, it is likely that better

232 performance in both memory and speed can be achieved by an indexing strategy designed

233 specifically for comparative metagenomic assembly, and we plan to explore such strategies in future

234 work. Furthermore, comparative assembly provides new opportunities for the development of

235 sequence alignment approaches that optimize the combined time of index creation and alignment.

236 Most of the recent developments in sequence alignment have assumed index construction to be a one-

237 time off-line operation, trading off a computationally intensive indexing approach for more efficient 238 queries.

\section{Conclusion}

240 We have described MetaCompass, a computational pipeline for comparative metagenomic assembly.

241 This novel method for metagenomic assembly leverages the increasing number of genome sequences

242 available in public databases. We have shown that comparative and de novo assemblies provide 
243 complementary strengths, and that combining both approaches effectively improves the overall

244 assembly, providing a consistent increase in the quality of the assembly. Even when distant reference

245 genomes are recruited, MetaCompass is competitive with de novo genome assembly methods. These

246 results are due to two critical steps. First, reference bias is avoided by constructing the consensus

247 sequence from the reads within the sample, using the reference genome as just a guide, and by

248 breaking the assembly where the reads indicate a structural disagreement with the reference. Second,

249 unmapped reads are used in a de novo assembly process to reconstruct the sections of the

250 metagenomic sample that are not similar to known reference genomes. In summary, we believe that

251 reference-guided approaches such as MetaCompass, will increasingly replace the more

252 computationally expensive and error-prone de novo assembly approaches as the collection of

253 available reference genome sequences increases.

\section{Methods}

256 Methods overview. First, we use MetaPhyler [13] to identify reference genomes that are most

257 closely related to the data represented in the input a sample. We use the NCBI RefSeq genome

258 database (June 2016) as the standard reference collection for MetaCompass. We only retain for

259 further consideration the genomes estimated by MetaPhyler to be represented at sufficient depth of

260 coverage. These genomes are aligned using Bowtie2 [42] (v2.2.9). The resulting read alignments are

261 then used to identify a minimal set of genomes that best explain all read alignments, then the read

262 alignments are used to construct contigs. We developed the tool buildcontig to generate a consensus

263 sequence for the contigs and then use Pilon [43] (v1.18) to correct the contigs in a way that reflects

264 the genome being assembled and to avoid biasing the reconstruction towards the reference sequence.

265 Contigs may be broken at this stage if the metagenomic sequence diverges from the reference 
sequence. Finally the reads that were not included in the reference-guided process outlined above are assembled using MEGAHIT [32] (v1.0.6) to reconstruct the metagenomic segments not represented in the reference collection. The details of each analysis step are described below.

Selecting reference genomes. While comparative assembly approaches have already been described

270 for single genomes [23,44] their use in metagenomic data is complicated by the multiple unknown

271 organisms and the thousands of genomes available in public databases. Building efficient indexes for

272 large reference collections is computationally challenging for short read aligners [41], both in term of

273 speed and memory consumption. For assembly, however we only need to use the genomes that are

274 detected in a sample. To speed up the genome reference selection step, we reduce the 31 marker

275 genes in MetaPhyler to 18 universally conserved marker genes in bacteria and archaea (intersection

276 between the sets of genes used by FetchMG [45, 46] and MetaPhyler [13]). The MetaPhyler index is

277 much smaller than a whole-genome index, yet still allows us to identify the closest reference genome

278 detected in the sample being assembled. We further speed up the execution of MetaPhyler by

279 restricting the analysis to just those reads that share at least one 28-mer with one of the marker gene

280 sequences in the database. We rely on kmer-mask

281 (http://kmer.sourceforge.net/wiki/index.php?Main_Page) to execute this filtering step. The selected

282 reads are then aligned to the marker collection using BLASTN with the parameters '-word_size 28 -

283 evalue 1e-10 -perc_identity 95 -max_target_seqs 100' and a minimum HSP alignment length of 35.

284 Since closely related genomes can share the same marker genes, we retain all hits with a bit score

285 equal to that of the top hit. Finally, we exclude from further consideration all the genomes with an

286 estimated coverage below a user-selected coverage threshold (2-fold, by default).

287 Aligning reads to reference sequences. The results presented in the paper are based on aligning the 288 reads to the selected reference genomes with Bowtie 2 [42] (parameters: --sam-nohead --sam-nosq -end-to-end --quiet --all -p 12). The alignments are then filtered to keep ties of lowest edit distance for 
each reads, allowing a read to be aligned in multiple locations similar to the best-strata option of

291 bowtie1.

292 Selecting a minimal reference set. In its simplest form, the comparative assembly approach involves

293 mapping the reads to a genome and using their relative placement within this genome to guide the

294 construction of contigs [23]. In the context of metagenomic data, however, this process is

295 complicated by the fact that individual reads may map to multiple reference genomes, some of which

296 are highly similar to each other. Adequately dealing with this ambiguity is critical for effective

297 assembly. If all read mappings are retained, allowing a read to be associated with multiple reference

298 genomes, the resulting assembly will be redundant, reconstructing multiple copies of the homologous

299 genomic regions. If for each read a random placement is selected from among the multiple

300 equivalent matches, none of the related genomes may recruit enough reads to allow assembly,

301 thereby leading to a fragmented reconstruction. Assigning reads to genomes according to their

302 estimated representation in the sample (determined, e.g., based on the number of reads uniquely

303 mapped to each genome), may bias the reconstruction towards the more divergent reference genomes,

304 which may lead to an overall poorer reconstruction of the genomic regions shared across related

305 genomes. Here we propose a parsimony-driven approach - identifying the minimal set of reference

306 genomes that explains all read alignments.

307 Formally, this problem can be framed as a set cover problem, an optimization problem which is NP-

308 hard. To solve this problem, we use a greedy approximation algorithm, which iteratively picks the set

309 of genomes that covers the greatest number of unused reads. It can be shown that this greedy

310 algorithm is the best-possible polynomial time approximation algorithm for the set cover problem

311 [47]. 
312 Building contigs. Given a set of reference genomes, selected as described above, a set of shotgun

313 reads, and the alignment between each read and reference genome, the process of creating contigs is

314 straightforward. For each nucleotide in each reference genome, we look at the bases from the reads

315 that are mapped to each locus, and pick the variant (nucleotide or indel) with the highest depth of

316 coverage as the consensus and report it. Minimum depth of coverage and length for creating contigs

317 can be specified through the program command-line options.

318 Removing reference-bias with Pilon. Differences between the sequences being assembled and the

319 reference genome used by MetaCompass can degrade the performance of the comparative assembly

320 process. We employ Pilon [43] to "polish" the reference-guided assemblies, thereby changing the

321 consensus sequence to resemble the data in the sample rather than the reference genome. During this

322 process we also identify signatures of larger differences between the metagenomic sample and the

323 reference sequence, and break the assembly at those locations.

324 Combining reference-guided and de novo assembly. We employ the de novo assembler MEGAHIT

325 to assemble reads that were unable to be mapped back to the reference-guided assembly generated by

326 MetaCompass. These reads represent microbes that are missing from our reference database and

327 novel variants. This approach allows the final assembly to capture both reference and non-reference

328 sequences. We chose MEGAHIT because it is currently the most efficient de novo assembler for

329 metagenomics [48]. MEGAHIT is also the default assembly methods for the JGI metagenomic

330 pipeline [49] and performed well in a recent review [50].

331 Gene prediction and marker gene detection. The genes were predicted in the contigs using

332 MetaGeneMark [51](v3.26) with the "MetaGeneMark_v1.mod" model parameter file and using the

333 option "-n" to remove partial genes containing long strings of " $\mathrm{N}$ ". The completion status of the 
334 genes (complete, lack 5', lack 3' and lack both) was defined by detecting all the common start codon

335 (“ATG”, “TTG”, “GTG”) and stop codon (“TAA”, “TAG”, “TGA”) of prokaryotic genes.

336 The 40 universal single copy marker proteins $[52,53]$ were identified in predicted genes using the

337 standalone version of fetchMG (v1.0) http://www.bork.embl.de/software/mOTU/) [46].

338 MetaQuast validation parameters. The command used to run MetaQuast was: 'metaquast.py -R

339 ./shakya_references --fragmented --gene-finding'

340 Synthetic metagenome assembly parameters. IDBA-UD requires a single fasta file that was

341 generated using the IDBA 'fq2fa --merge --filter' command. MEGAHIT was run using the options '--

342 presets meta-sensitive --min-count 3 --min-contig-len 300 -t 12'. MetaSPAdes was run using the

343 options '--meta -t 12', then all contigs shorter than 300 nt and with less than $3 \mathrm{X}$ coverage were

344 removed. IDBA-UD was run using the options '--min_count 3 --min_contig 300 --mink 20 --maxk

345100 --num_threads 12'. MetaCompass was run using the options -m [1,2,3] -g 300 -t 16' on the

346 synthetic dataset and '-m 3 -g 300 -t 16' on the HMP2 samples.

347 Data availability. A list of all available HMP samples was obtained by combining those available

348 from the HMP Data Analysis and Coordination Center (DACC) (www.hmpdacc.org) and the HMP

349 SRA project PRJNA48479 on 11/16/2016. Any sample listed in the SRA and not in the DACC was

350 downloaded and processed by the HMP WGS Read Processing Protocol

351 (http://www.hmpdacc.org/doc/ReadProcessing_SOP.pdf). Three DACC samples were corrupt or

352 extracted to a duplicate SRS identifier (SRS023176, SRS043422, and SRS057182) and were

353 downloaded from SRA and processed as above. A total of 98454 samples were excluded from the

354 downloaded set. This resulted in 2,713 samples. Some samples (504) had no references recruited and

355 were excluded from further analysis. This resulted in 2,209 MetaCompass assemblies. All HMP2

356 assemblies available at ftp://public-ftp.hmpdacc.org/HMASM/IDBA/ were downloaded (2,341 total 
assemblies). A total of 2,077 samples (Supplementary Table 4) had both an HMP2 assembly and a MetaCompass assembly and were used for the analysis.

360 The set of known genomes for the synthetic dataset is available via the Supplementary Table 2 from

361 Shakya et al [31].

362 Software availability. MetaCompass is available as an open-source package at:

363 https:/github.com/marbl/MetaCompass. The code is licensed under the Artistic License 2.0:

364 https://opensource.org/licenses/Artistic-2.0

\section{List of abbreviations}

367 DACC - Data Analysis and Coordination Center

368 HMP - Human Microbiome Project

369 NCBI - National Center for Biotechnology

370 RefSeq - NCBI Reference Sequence Database

371 SRA - Short read archive

\section{$373 \quad$ Funding}

374 The authors were supported in part by the NIH, grants R01-HG-004885 and R01-AI-100947, by the

375 NSF, grants IIS-1117247 and IIS-0812111, and the Office of Naval Research under cooperative

376 agreement number N00173162C001, all to MP. SK was supported by the Intramural Research 
377 Program of the National Human Genome Research Institute, National Institutes of Health. SK has

378 received funding for travel and accommodation expenses in order to speak at Oxford Nanopore

379 Technologies conferences.

\section{Author's contributions}

BL and MP designed the approach. VC, BL, TT, and MA implemented the algorithms, methods, and

383 scripts described in the paper. VC, TT, and SK generated the assemblies presented in the paper. TT,

384 VC, and MA validated the assembly results and performed the comparisons between different

385 assemblers. VC, MP, BL, TT and wrote the paper. All authors were involved in reviewing and

386 revising the manuscript. All authors read and approved the manuscript.

\section{Acknowledgments}

389 We would like to thank Owen White, Anup Mahurkar, and other members of the HMP DACC for

390 helping us make public the revised assemblies of the HMP data. We thank C. Titus Brown and the

391 other reviewers for their thoughtful reviews.

393 References

394 1. Hooper L V. Commensal Host-Bacterial Relationships in the Gut. Science (80- ). 2001;292:1115-

395 8. doi:10.1126/science. 1058709.

396 2. Tringe SG, Rubin EM. Metagenomics: DNA sequencing of environmental samples. Nat Rev

397 Genet. 2005;6:805-14. doi:10.1038/nrg1709. 
3. Qin J, Li R, Raes J, Arumugam M, Burgdorf KS, Manichanh C, et al. A human gut microbial gene doi:10.1038/nature08821. human microbiome research. Nature. 2012;486:215-21. doi:10.1038/nature11209.

5. Kingsford C, Schatz MC, Pop M. Assembly complexity of prokaryotic genomes using short reads.

BMC Bioinformatics. 2010;11:21. doi:10.1186/1471-2105-11-21.

6. Laserson J, Jojic V, Koller D. Genovo: De Novo Assembly for Metagenomes. J Comput Biol. 2011;18:429-43. doi:10.1089/cmb.2010.0244.

7. Zerbino DR, Birney E. Velvet: algorithms for de novo short read assembly using de Bruijn graphs.

Genome Res. 2008;18:821-9. doi:10.1101/gr.074492.107.

8. Peng Y, Leung HCM, Yiu SM, Chin FYL. IDBA-UD: a de novo assembler for single-cell and

410 metagenomic sequencing data with highly uneven depth. Bioinformatics. 2012;28:1420-8.

411 doi:10.1093/bioinformatics/bts174.

412 9. Simpson JT, Wong K, Jackman SD, Schein JE, Jones SJM, Birol I. ABySS: a parallel assembler

413 for short read sequence data. Genome Res. 2009;19:1117-23. doi:10.1101/gr.089532.108.

414 10. Li R, Zhu H, Ruan J, Qian W, Fang X, Shi Z, et al. De novo assembly of human genomes with

415 massively parallel short read sequencing. Genome Res. 2010;20:265-72. doi:10.1101/gr.097261.109.

416 11. Rho M, Tang H, Ye Y. FragGeneScan: predicting genes in short and error-prone reads. Nucleic

417 Acids Res. 2010;38:e191. doi:10.1093/nar/gkq747.

418 12. Brady A, Salzberg SL. Phymm and PhymmBL: metagenomic phylogenetic classification with

419 interpolated Markov models. Nat Methods. 2009;6:673-6. doi:10.1038/nmeth.1358. 
420 13. Liu B, Gibbons T, Ghodsi M, Treangen T, Pop M. Accurate and fast estimation of taxonomic

421 profiles from metagenomic shotgun sequences. BMC Genomics. 2011;12 Suppl 2:S4.

422 doi:10.1186/1471-2164-12-S2-S4.

423 14. Segata N, Waldron L, Ballarini A, Narasimhan V, Jousson O, Huttenhower C. Metagenomic

424 microbial community profiling using unique clade-specific marker genes. Nat Methods. 2012;9:811-

425 4. doi:10.1038/nmeth.2066.

426 15. Abubucker S, Segata N, Goll J, Schubert AM, Izard J, Cantarel BL, et al. Metabolic

427 reconstruction for metagenomic data and its application to the human microbiome. PLoS Comput

428 Biol. 2012;8:e1002358. doi:10.1371/journal.pcbi.1002358.

429 16. Menzel P, Ng KL, Krogh A, Marth G, Lipman D. Fast and sensitive taxonomic classification for 430 metagenomics with Kaiju. Nat Commun. 2016;7:11257. doi:10.1038/ncomms11257.

431 17. Chin C-S, Alexander DH, Marks P, Klammer AA, Drake J, Heiner C, et al. Nonhybrid, finished 432 microbial genome assemblies from long-read SMRT sequencing data. Nat Methods. 2013;10:563-9. 433 doi:10.1038/nmeth.2474.

434 18. Koren S, Harhay GP, Smith TPL, Bono JL, Harhay DM, Mcvey SD, et al. Reducing assembly 435 complexity of microbial genomes with single-molecule sequencing. Genome Biol. 2013;14:R101. 436 doi:10.1186/gb-2013-14-9-r101.

19. Koren S, Phillippy AM. One chromosome, one contig: complete microbial genomes from long-

438 read sequencing and assembly. Curr Opin Microbiol. 2015;23:110-20.

439 doi:10.1016/j.mib.2014.11.014.

440 20. Frank JA, Pan Y, Tooming-Klunderud A, Eijsink VGH, McHardy AC, Nederbragt AJ, et al.

441 Improved metagenome assemblies and taxonomic binning using long-read circular consensus 
sequence data. Sci Rep. 2016;6:25373. doi:10.1038/srep25373.

21. Driscoll CB, Otten TG, Brown NM, Dreher TW. Towards long-read metagenomics: complete

444 assembly of three novel genomes from bacteria dependent on a diazotrophic cyanobacterium in a

445 freshwater lake co-culture. Stand Genomic Sci. 2017;12:9. doi:10.1186/s40793-017-0224-8.

446 22. Husemann P, Stoye J. r2cat: synteny plots and comparative assembly. Bioinformatics.

447 2010;26:570-1. doi:10.1093/bioinformatics/btp690.

448 23. Pop M, Phillippy A, Delcher AL, Salzberg SL. Comparative genome assembly. Brief Bioinform. 449 2004;5:237-48. doi:10.1093/bib/5.3.237.

450 24. O’Leary NA, Wright MW, Brister JR, Ciufo S, Haddad D, McVeigh R, et al. Reference sequence 451 (RefSeq) database at NCBI: current status, taxonomic expansion, and functional annotation. Nucleic

Acids Res. 2016;44:D733-45. doi:10.1093/nar/gkv1189.

25. Nutrition C for FS and A. Whole Genome Sequencing (WGS) Program - GenomeTrakr Network.

454 https://www.fda.gov/Food/FoodScienceResearch/WholeGenomeSequencingProgramWGS/ucm3631

455 34.htm. Accessed 2 Oct 2017.

26. Bankevich A, Nurk S, Antipov D, Gurevich AA, Dvorkin M, Kulikov AS, et al. SPAdes: A New

457 Genome Assembly Algorithm and Its Applications to Single-Cell Sequencing. J Comput Biol. 2012;19:455-77. doi:10.1089/cmb.2012.0021.

460 Genomes and Mini-metagenomes from Highly Chimeric Reads. Springer, Berlin, Heidelberg; 2013.

461 p. 158-70. doi:10.1007/978-3-642-37195-0_13.

462 28. Truong DT, Tett A, Pasolli E, Huttenhower C, Segata N. Microbial strain-level population

463 structure and genetic diversity from metagenomes. Genome Res. 2017;27:626-38. 
doi:10.1101/gr.216242.116.

29. Li J, Jia H, Cai X, Zhong H, Feng Q, Sunagawa S, et al. An integrated catalog of reference genes in the human gut microbiome. Nat Biotechnol. 2014;32:834-41. doi:10.1038/nbt.2942. and rRNA microbial diversity characterization using archaeal and bacterial synthetic communities. Environ Microbiol. 2013;15:1882-99. doi:10.1111/1462-2920.12086.

32. Li D, Liu C-M, Luo R, Sadakane K, Lam T-W. MEGAHIT: an ultra-fast single-node solution for 2015;31:1674-6. doi:10.1093/bioinformatics/btv033. assembler. Genome Res. 2017;:gr.213959.116. doi:10.1101/gr.213959.116. assemblies. Bioinformatics. 2013;29:1072-5. doi:10.1093/bioinformatics/btt086.

35. HMP2 assembly details. http://gembox.cbcb.umd.edu/metacompass. and dynamics in the expanded Human Microbiome Project. Nature. 2017;550:61. doi:10.1038/nature23889. 
38. Belkaid Y, Segre JA. Dialogue between skin microbiota and immunity. Science (80- ).

http://www.ncbi.nlm.nih.gov/pubmed/19396949. Accessed 6 May 2017.

495 alignments. Genome Biol. 2014;15:R46. doi:10.1186/gb-2014-15-3-r46. 
508 47. Feige U. A threshold of $\ln \mathrm{n}$ for approximating set cover. J ACM. 1998;45:634-52.

509 doi:10.1145/285055.285059.

510 48. Olson ND, Treangen TJ, Hill CM, Cepeda-Espinoza V, Ghurye J, Koren S, et al. Metagenomic

511 assembly through the lens of validation: recent advances in assessing and improving the quality of

512 genomes assembled from metagenomes. Brief Bioinform. 2017. doi:10.1093/bib/bbx098.

513 49. Clum A. Genome Assembly at JGI. Genomic Technologies Workshop JGI User Meeting. 2015.

514 http://3q8i7m48ig9en9v121qx83t166i.wpengine.netdna-cdn.com/wp-

515 content/uploads/sites/2/2015/04/04_Alicia_2015.03.23.JGI-user-meeting-GT-workshop-Assembly-

516 talk-Clum-FINAL.pdf. Accessed 13 Oct 2017.

517 50. Greenwald WW, Klitgord N, Seguritan V, Yooseph S, Venter JC, Garner C, et al. Utilization of 518 defined microbial communities enables effective evaluation of meta-genomic assemblies. BMC

519 Genomics. 2017;18:296. doi:10.1186/s12864-017-3679-5.

520 51. Zhu W, Lomsadze A, Borodovsky M. Ab initio gene identification in metagenomic sequences.

$521 \quad$ Nucleic Acids Res. 2010;38:e132. doi:10.1093/nar/gkq275.

522 52. Ciccarelli FD, Doerks T, von Mering C, Creevey CJ, Snel B, Bork P. Toward automatic

523 reconstruction of a highly resolved tree of life. Science. 2006;311:1283-7.

524 doi:10.1126/science.1123061.

525 53. Sorek R, Zhu Y, Creevey CJ, Francino MP, Bork P, Rubin EM. Genome-wide experimental

526 determination of barriers to horizontal gene transfer. Science. 2007;318:1449-52.

527 doi:10.1126/science. 1147112.

528 54. Mikheenko A, Saveliev V, Gurevich A. MetaQUAST: evaluation of metagenome assemblies.

529 Bioinformatics. 2016;32:1088-90. doi:10.1093/bioinformatics/btv697. 
bioRxiv preprint doi: https://doi.org/10.1101/212506; this version posted November 1,2017 . The copyright holder for this preprint (which was not certified by peer review) is the author/funder, who has granted bioRxiv a license to display the preprint in perpetuity. It is made available under aCC-BY 4.0 International license.

530 55. Li K, Bihan M, Yooseph S, Methé BA, Ludwig W. Analyses of the Microbial Diversity across

531 the Human Microbiome. PLoS One. 2012;7:e32118. doi:10.1371/journal.pone.0032118.

532

533

534 
535 Table 1. Evaluation of performance on synthetic dataset. MetaCompass (X) indicates the

536 minimum coverage setting (1X or 2X), and MetaCompass.nr indicates all 64 reference genomes

537 comprising the Shakya et al. dataset were removed from the database. Tool indicates the assembler, \#

538 ctgs the total number of assembled contigs reported by each assembler, Max ctg is the maximum

539 contig length (broken at errors) for all assembled contigs, Median Genome Recovery (\%) is the

540 median percentage of each of the synthetic genomes that is recovered, Complete Marker Genes

541 (median) is the median number of fully reconstructed marker genes, Total Aligned Length is the

542 sum of the length of contigs aligned to the reference genomes, Total Unaligned Length is the sum of

543 the length of unaligned contigs, and MetaQuast reported errors are error statistics generated with

544 MetaQuast.

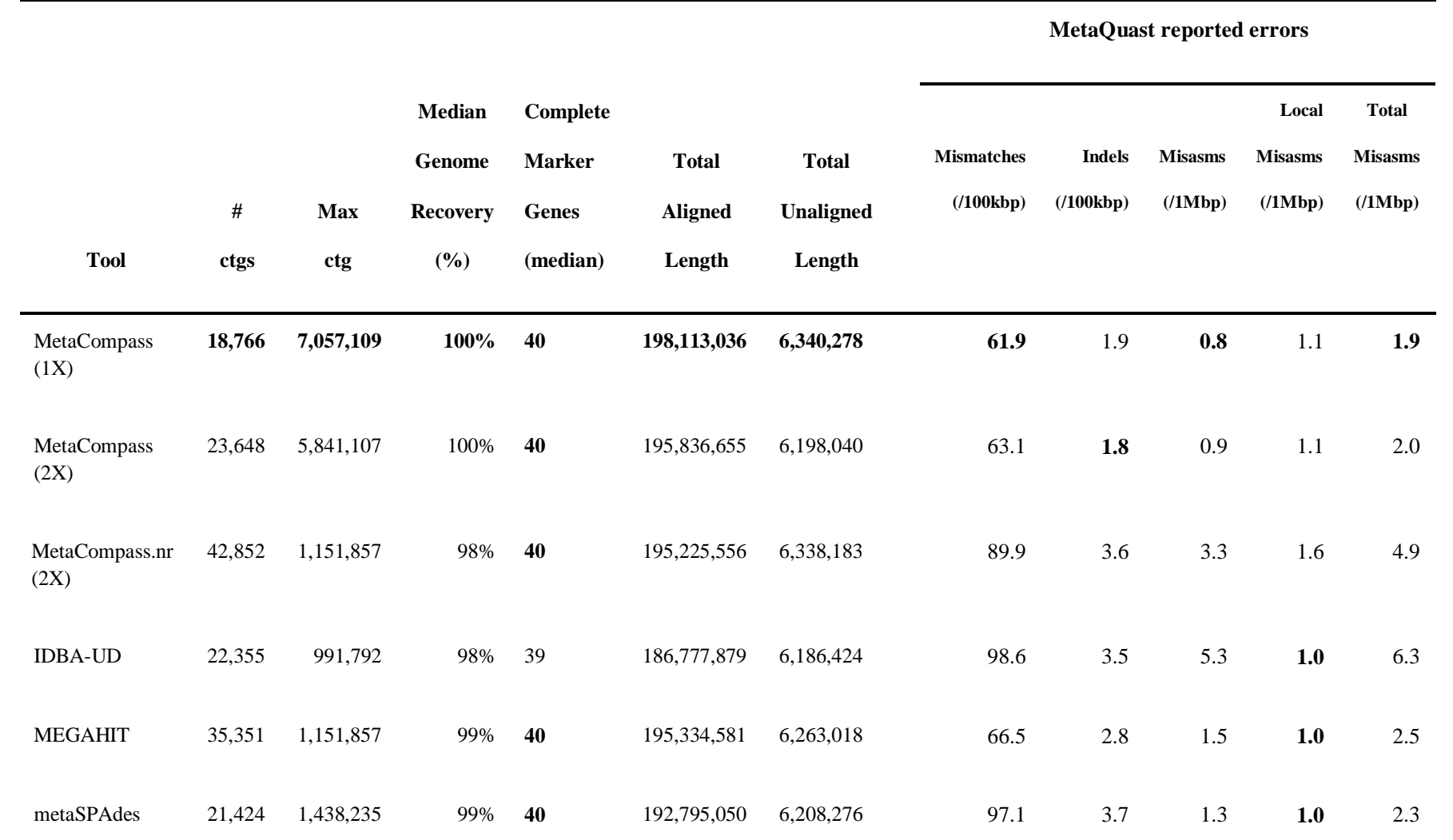


547 Table 2. Evaluation of performance on down-sampled synthetic dataset. The synthetic dataset

548 was down-sampled to only contain $10 \%$ of the total reads. Tool indicates the assembler, \# ctgs the

549 total number of assembled contigs reported by each assembler, Max ctg is the maximum contig

550 length (broken at errors) of all assembled contigs, Median Genome Recovery (\%) is the median

551 percentage of each of the synthetic genomes that is recovered, Complete Marker Genes (median) is

552 the median number of fully reconstructed marker genes, Total Aligned Length is the sum of the

553 length of contigs aligned to the truth genomes, Total Unaligned Length is the sum of the length of

554 unaligned contigs, and MetaQuast reported errors are error statistics generated with MetaQuast

$555[54]$.

556

\begin{tabular}{|c|c|c|c|c|c|c|c|c|c|c|}
\hline & & & & \multirow[b]{2}{*}{ Complete } & & & \multicolumn{4}{|c|}{ MetaQuast reported errors } \\
\hline & & & Median & & Total & Tata & $\begin{array}{c}\text { Mismatches } \\
\text { (/100kbp) }\end{array}$ & $\begin{array}{r}\text { Indels } \\
\text { (/100kbp) }\end{array}$ & $\begin{array}{l}\text { Misasms } \\
\text { (>1 kbp) }\end{array}$ & $\begin{array}{l}\text { Misasms } \\
(<1 \mathrm{kbp})\end{array}$ \\
\hline & $\#$ & Max & Recovery & Genes & Aligned & Unaligned & & & & \\
\hline Method & ctgs & Ctg & $(\%)$ & (median) & Length & Length & & & & \\
\hline MetaCompass & 71457 & 962,929 & $90 \%$ & 22 & $134,008,055$ & $3,009,931$ & 117.6 & 1.9 & 112 & 33 \\
\hline IDBA-UD & 43973 & 120159 & $45 \%$ & 6 & $75,970,693$ & $1,564,008$ & 175.0 & 5.3 & 3447 & 93 \\
\hline MEGAHIT & 62842 & 209,706 & $76 \%$ & 15 & $105,665,678$ & $2,774,432$ & 128.0 & 4.1 & 772 & 122 \\
\hline metaSPAdes & 67138 & 287,554 & $80 \%$ & 16 & $111,636,826$ & $3,154,199$ & 133.0 & 4.3 & 470 & 115 \\
\hline
\end{tabular}


Table 3. Re-assembly of 2,077 samples generated in the Human Microbiome Project. The results are aggregated by body site. \# indicates the total reads per sample, Avg cvg per sample $(\mathbf{X})$ is the mean estimate read coverage calculated based on the de novo assembly of each sample and body site,

561 Shannon Entropy (median) is the Shannon diversity value per body site as reported in Li et al 2012

562 [55].The rows labeled MC contain results obtained with MetaCompass. The rows labeled HMP2

563 show the statistics for contigs from the production HMP2 assembly. Total Size (Mbp) is the total

564 assembly size for each method, Max ctg size (kbp) is the size of the largest contig, Median

565 Size@1Mbp (kbp) represents the mediad size of the largest contig C such that the sum of all contigs

566 larger than $\mathrm{C}$ exceeds $1 \mathrm{Mbp}$. Median Complete Genes represents the median number of complete

567 genes per sample. Median Marker Genes indicates the median number of complete marker genes

568 per sample.

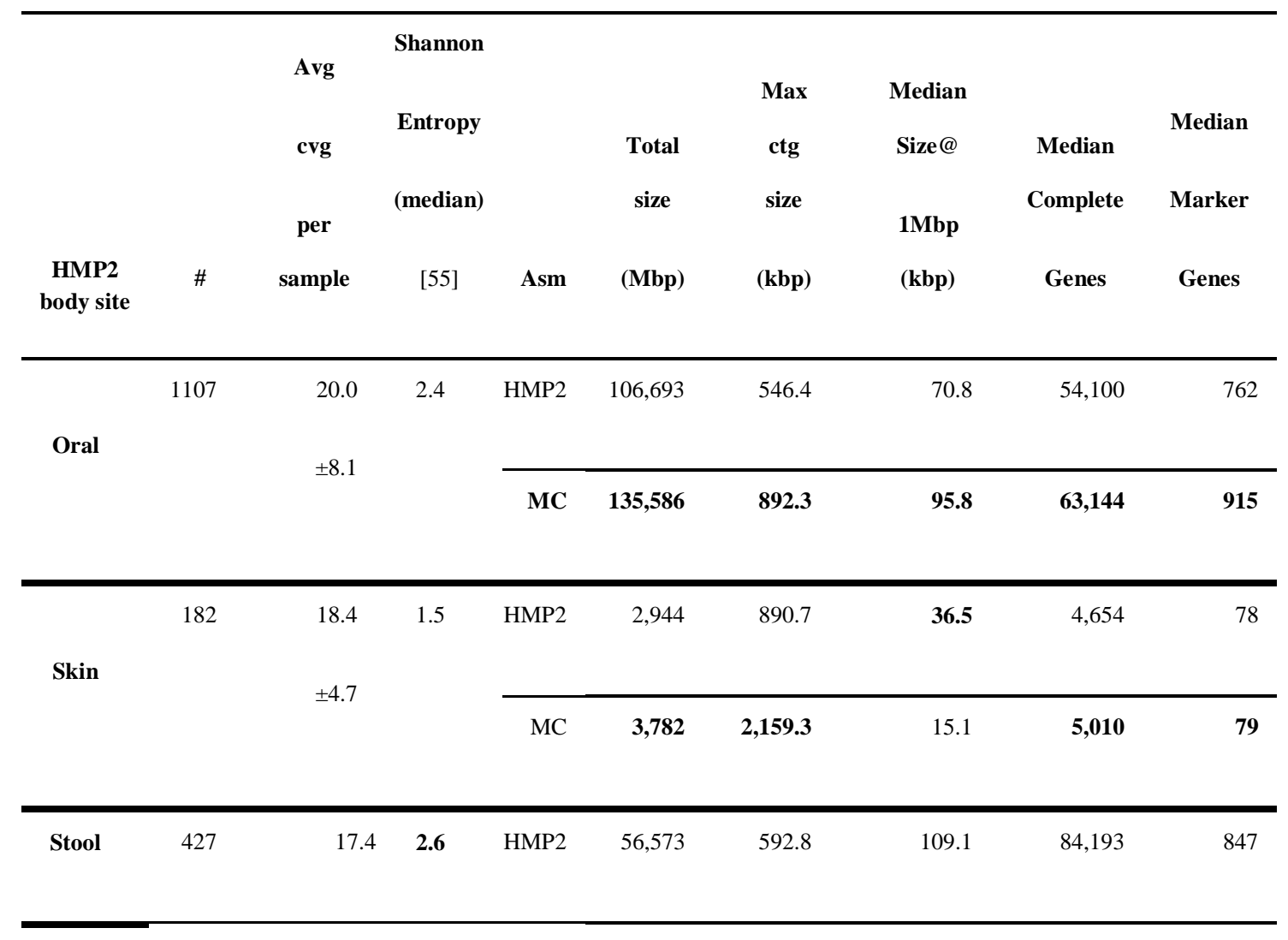


bioRxiv preprint doi: https://doi org/10.1101/212506 this version posted November 1 2017. The copyright holder for this preprint (which was not certified by peer review) is the author/funder, who has granted bioRxiv a license to display the preprint in perpetuity. It is made available under aCC-BY 4.0 International license.

\begin{tabular}{llllllll}
\hline \pm 4.9 & MC & $\mathbf{6 6 , 8 3 8}$ & $\mathbf{3 , 3 0 1 . 0}$ & $\mathbf{2 3 0 . 9}$ & $\mathbf{9 4 , 2 9 7}$ & 1,043
\end{tabular}

\begin{tabular}{|c|c|c|c|c|c|c|c|c|c|}
\hline & 159 & 7.8 & 0.2 & HMP2 & 1,179 & 465.8 & 28.7 & 2,539 & 45 \\
\hline \multicolumn{10}{|l|}{ Vaginal } \\
\hline & & & & MC & 1,458 & 558.0 & 16.1 & 2,934 & 60 \\
\hline \multicolumn{10}{|c|}{184,518} \\
\hline$(+\mathbf{N A})$ & $(202)$ & & & MC & 232,161 & $3,301.0$ & 114.6 & 57,639 & 764 \\
\hline
\end{tabular}


571 Table 4. Results of Human Microbiome Project analysis within the reference genomes. The

572 results are aggregated by body site. \# indicates the total reads per sample, Avg cvg per sample (X) is

573 the mean estimate read coverage calculated based on the de novo assembly of each sample and body

574 site, Shannon Entropy (median) is the Shannon diversity value per body site as reported in Li et al

5752012 [55].The rows labeled MC contain results obtained with MetaCompass. The rows labeled

576 HMP2 show the statistics for contigs from the production HMP2 assembly. Total Size (Mbp) is the

577 total assembly size for each method, Max ctg size (kbp) is the size of the largest contig, Median

578 Size@1Mbp (kbp) represents the median size of the largest contig C such that the sum of all contigs

579 larger than C exceeds 1Mbp. Median Complete Genes represents the median number of complete

580 genes per sample. Median Marker Genes indicates the median number of complete marker genes

581 per sample.

\begin{tabular}{|c|c|c|c|c|c|c|c|c|c|}
\hline \multirow[b]{4}{*}{$\begin{array}{c}\text { HMP2 } \\
\text { body site }\end{array}$} & \multirow[b]{4}{*}{$\#$} & \multirow{2}{*}{$\begin{array}{l}\text { Avg } \\
\text { cvg }\end{array}$} & \multicolumn{3}{|l|}{ Shannon } & & \multirow{3}{*}{$\begin{array}{c}\text { Median } \\
\text { Complete }\end{array}$} & \multirow{3}{*}{$\begin{array}{l}\text { Median } \\
\text { Marker }\end{array}$} \\
\hline & & & Entropy & & Total & ctg & Size@ & & \\
\hline & & per & (median) & & size & size & 1Mbp & & \\
\hline & & sample & {$[55]$} & Asm & (Mbp) & (kbp) & $(\mathbf{k b p})$ & Genes & Genes \\
\hline \multirow{3}{*}{ Oral } & 1107 & 20.0 & 2.4 & HMP2 & 10,977 & 162.2 & 10.1 & 5411 & 176 \\
\hline & & \pm 8.1 & & & & & & & \\
\hline & & & & MC & 17,731 & 594.0 & 23.4 & 8899 & 265 \\
\hline \multirow{3}{*}{ Skin } & 182 & 18.4 & 1.5 & HMP2 & 615 & 716.9 & 15.4 & 1973 & 35 \\
\hline & & +47 & & & & & & & \\
\hline & & & & $\mathrm{MC}$ & 618 & $2,159.3$ & 8.3 & 1652 & 35 \\
\hline Stool & 427 & 17.4 & 2.6 & HMP2 & 8,655 & 217.3 & 33.2 & 12512 & 142 \\
\hline
\end{tabular}




\begin{tabular}{llllllll}
\hline & \pm 4.9 & MC & $\mathbf{8 , 6 6 5}$ & $\mathbf{3 , 3 0 1 . 0}$ & $\mathbf{1 0 4 . 3}$ & $\mathbf{1 2 7 5 9}$ & 217
\end{tabular}

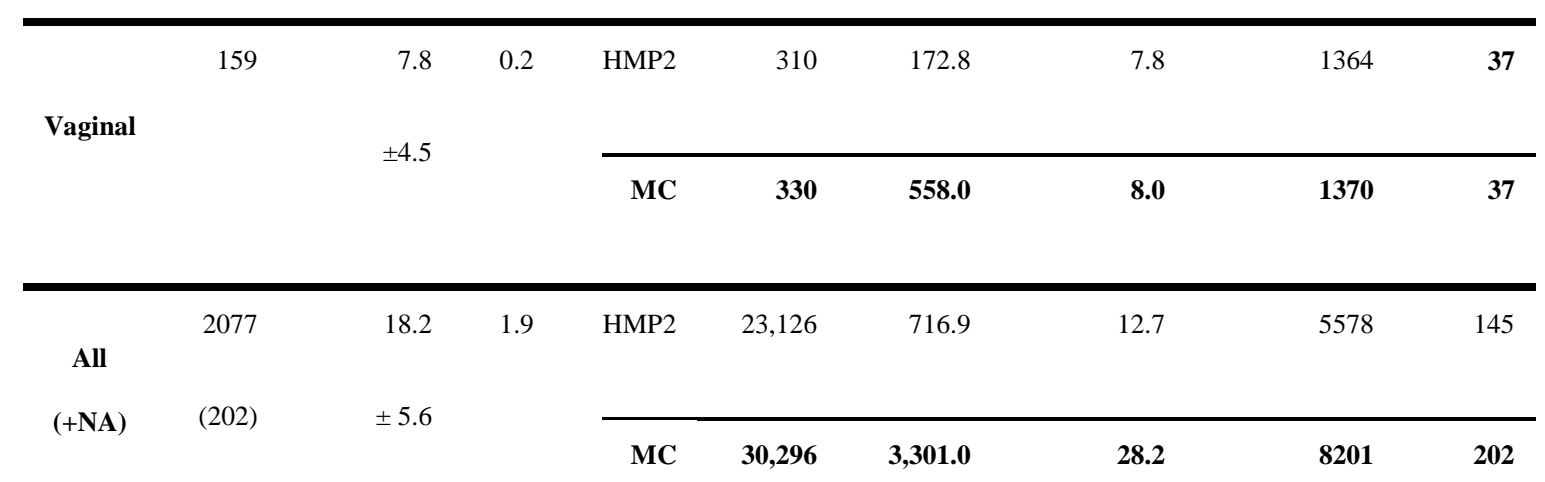

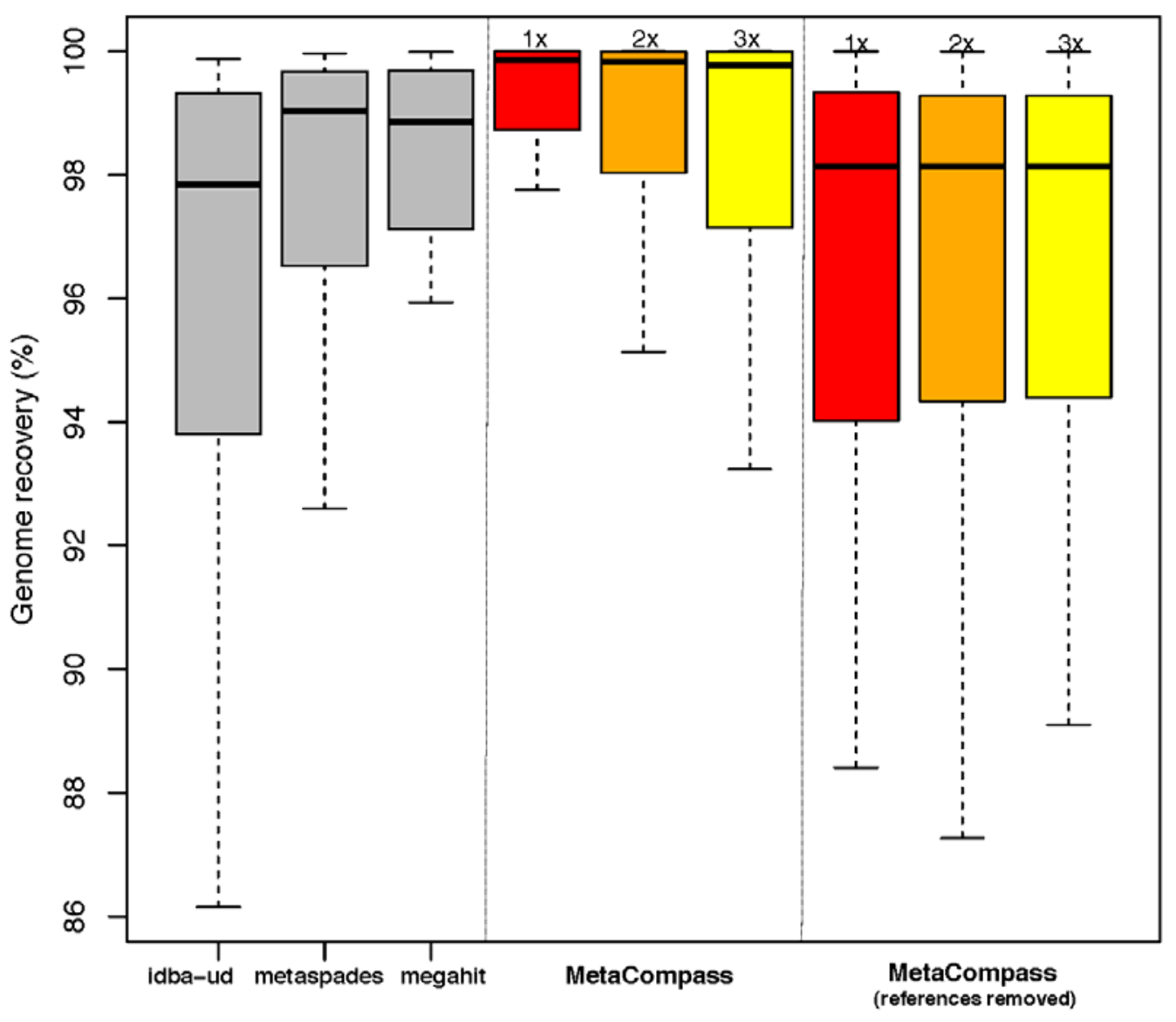

585 Figure 1. Genome recovery percentages in synthetic metagenome (MetaCompass versus de novo 
present in the synthetic metagenome). $\mathrm{x}$-axis indicates the assembly method, either IDBA-UD, metaSPAdes, MEGAHIT, or MetaCompass. MetaCompass was run both with the reference genomes present in the database (recruited as described in the methods) and without the truth reference

590 genomes in the database (they were individually removed). $y$-axis indicates the genome recovery

591 percentage, $0 \%$ indicates the genome was unassembled, whereas $100 \%$ indicates the genome was 592 fully assembled.

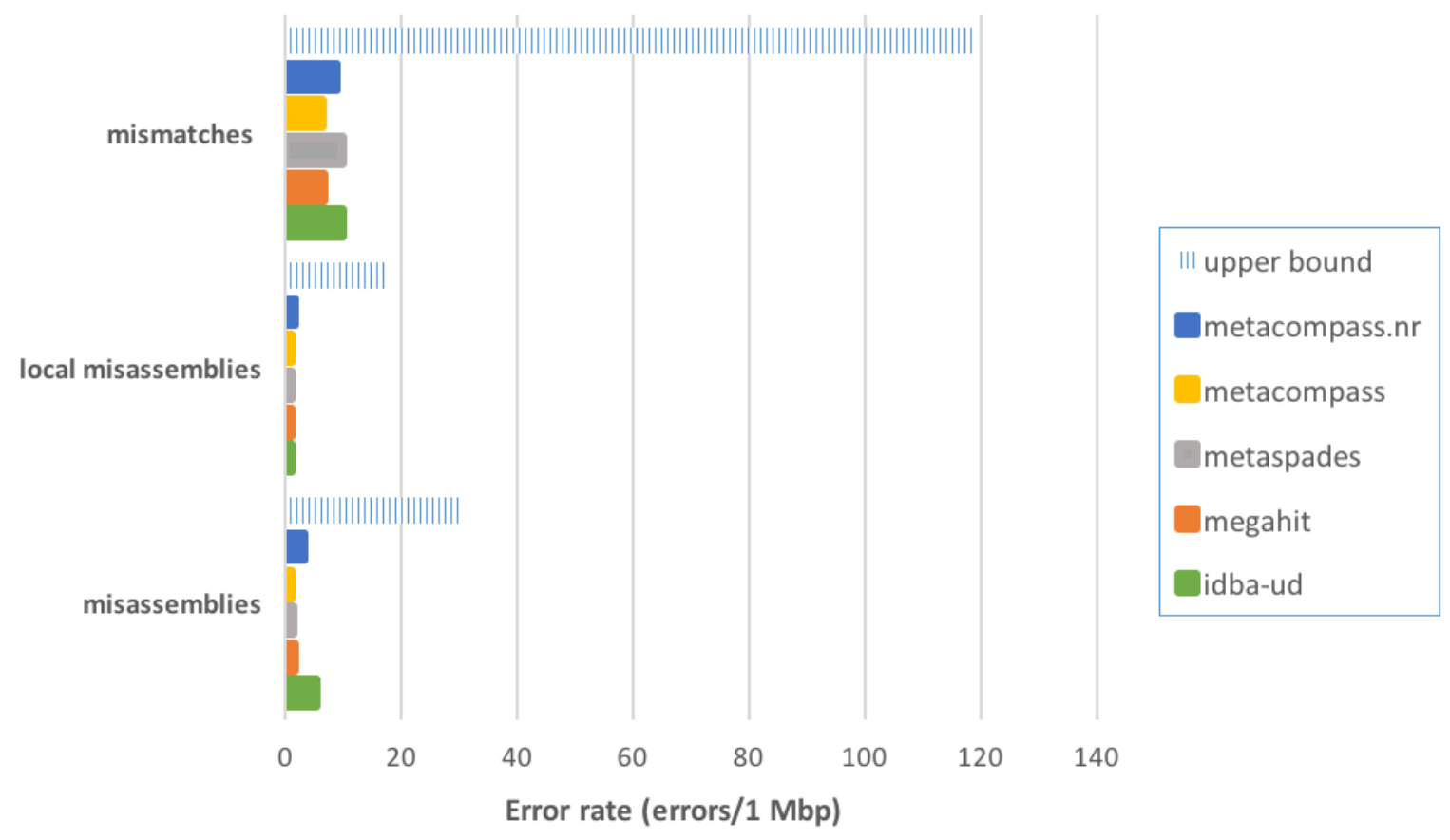

594 Figure 2. Error profile on synthetic dataset. The hashed blue bar represents the difference between the second-best reference genome (recruited by MetaCompass) and the true genome represented in

596 the sample. This bar can be viewed as an upper bound on the errors metacompass.nr could make if it

597 simply reconstructed the reference genome. Mismatches are the number of bases in a contig that

598 differ from the reference genome. Misassemblies include large-scale (left flanking region aligns $>1$

599 kbp away from right flanking region) relocations, interspecies relocations, translocations, and

600 inversions. Local misassemblies include small-scale (left flanking region aligns <=1 kbp away from 
601 right flanking region) translocations and inversions. All errors are normalized to represent rates per 1

602 Mbp.

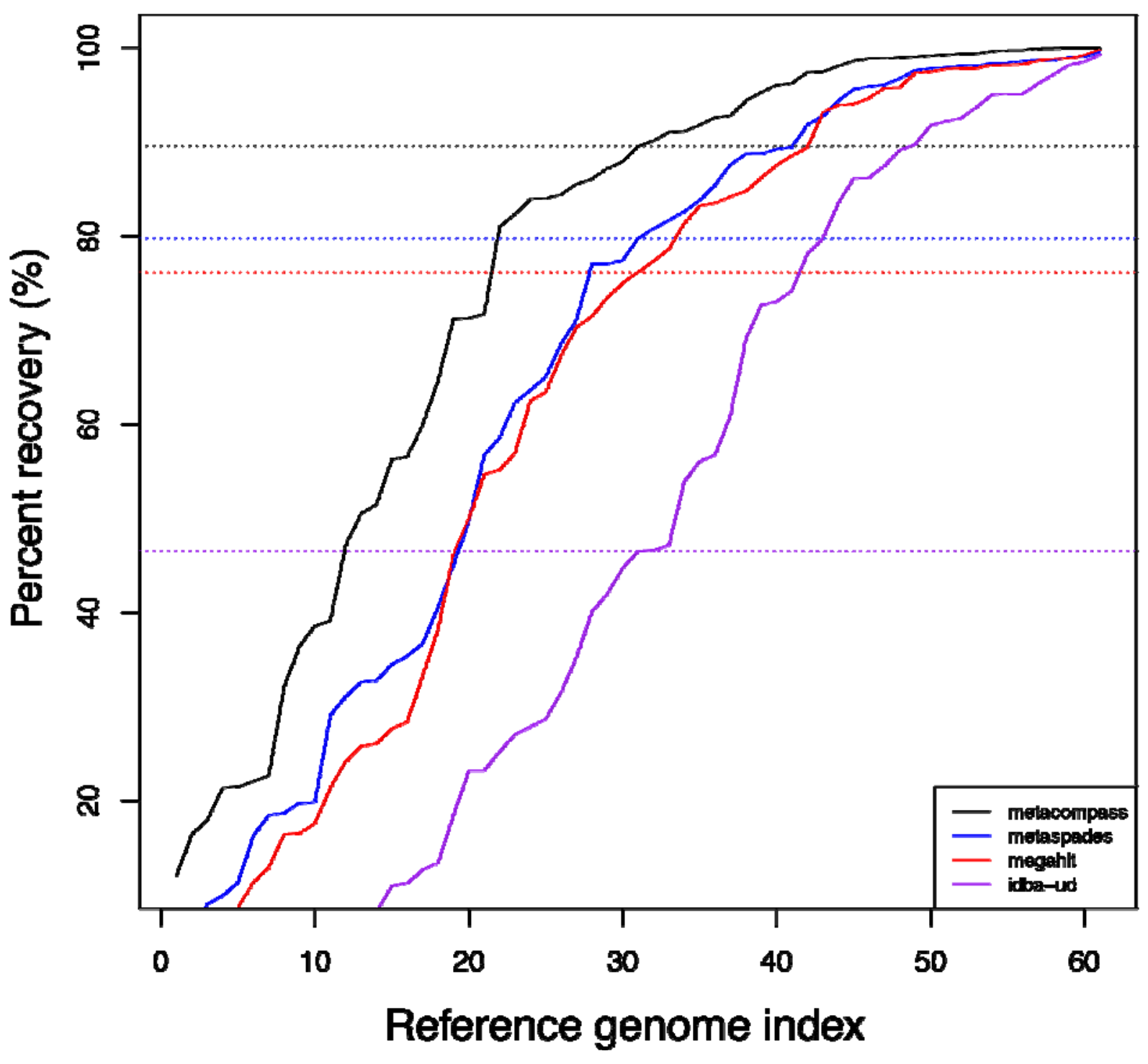

604 Figure 3. MetaCompass performance on low coverage dataset. Results obtained by down-

605 sampling the Shakya et al. synthetic genome to just $10 \%$ of the original set of reads. The 64 genomes

606 present in the sample are ordered per assembler by percent recovery, from lowest to highest. The y-

607 axis indicates how much of the $n$-th reference was covered by correctly assembled contigs (can range

608 from $0 \%$ to $100 \%$ ). The colored dashed lines indicate the median percent recovery for each

609 assembler. 

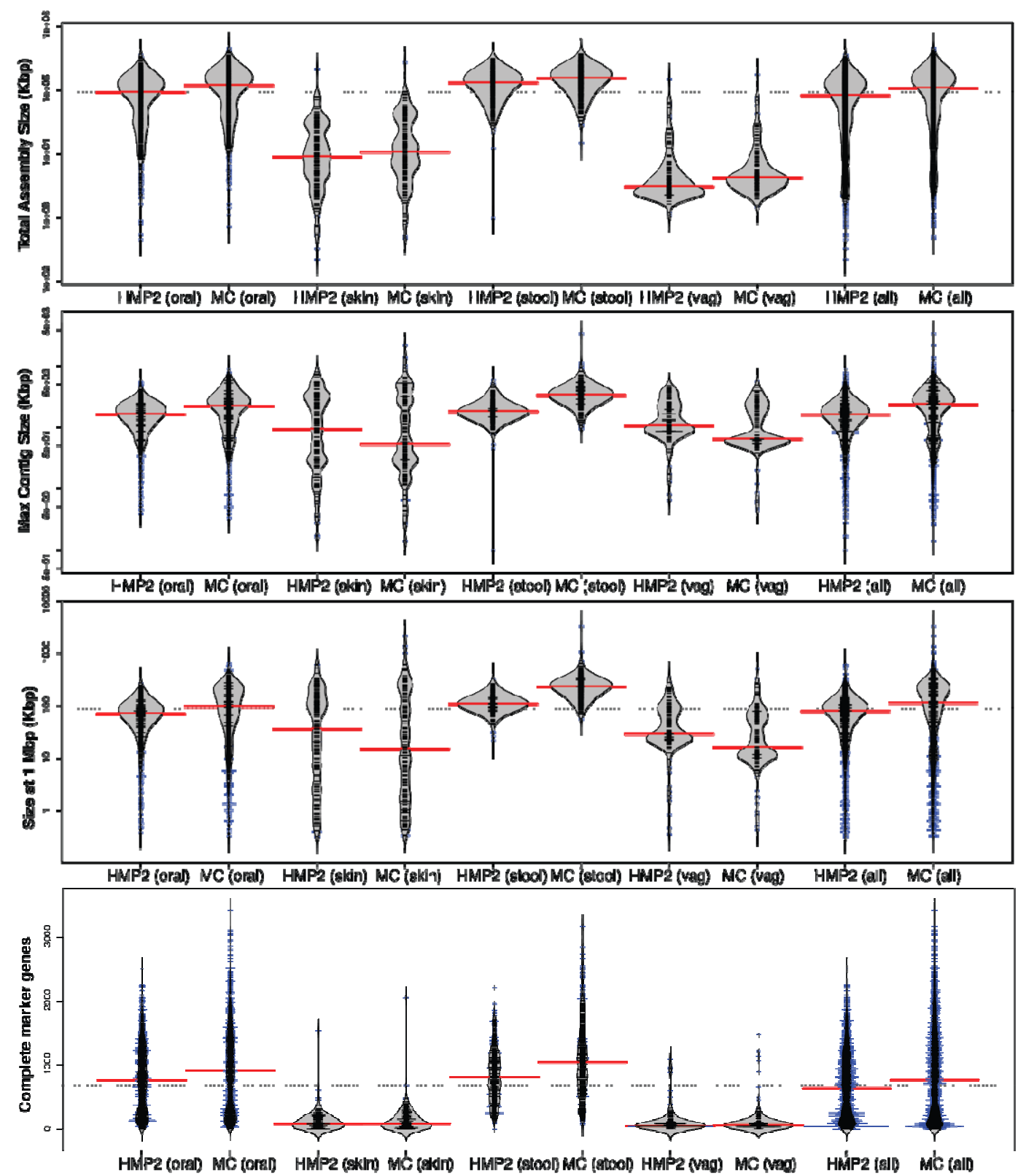

611 Figure 4. Comparative assembly of 2,077 metagenomic samples from the HMP2 Project. The

612 'bean plots' represent the distribution of assembly contiguity and completeness statistics across all

613 samples within the data. The $\mathrm{x}$ axis organizes the data by assembly and body site. The y-axis

614 indicates the statistic used to evaluate the assembly contiguity or completeness. The top panel shows

615 total assembly size (kbp), the second panel shows maximum contig size (kbp), the third panel shows

616 the size of the contig at $1 \mathrm{Mbp}$, and the bottom panel shows the complete marker genes assembled per

617 sample. 